\title{
A Synthesis of VIIRS Solar and Lunar Calibrations
}

\author{
Robert E. Eplee, Jr., ${ }^{a}$ Kevin R. Turpie, ${ }^{b}$ Gerhard Meister, ${ }^{c}$ Frederick S. Patt, ${ }^{a}$ \\ Gwyn F. Fireman, ${ }^{a}$ Bryan A. Franz, ${ }^{c}$ and Charles R. McClain ${ }^{c}$ \\ ${ }^{a}$ Science Applications International Corporation, Beltsville, Maryland 20705, USA \\ ${ }^{b}$ University of Maryland, Baltimore County, Catonsville, Maryland, 21250, USA \\ ${ }^{c}$ NASA Goddard Space Flight Center, Greenbelt, Maryland 20771, USA
}

\begin{abstract}
The NASA VIIRS Ocean Science Team (VOST) has developed two independent calibrations of the SNPP VIIRS moderate resolution reflective solar bands using solar diffuser and lunar observations through June 2013. Fits to the solar calibration time series show mean residuals per band of $0.078-0.10 \%$. There are apparent residual lunar libration correlations in the lunar calibration time series that are not accounted for by the ROLO photometric model of the Moon. Fits to the lunar time series that account for residual librations show mean residuals per band of $0.071-0.17 \%$. Comparison of the solar and lunar time series shows that the relative differences in the two calibrations are $0.12-0.31 \%$. Relative uncertainties in the VIIRS solar and lunar calibration time series are comparable to those achieved for SeaWiFS, Aqua MODIS, and Terra MODIS. Intercomparison of the VIIRS lunar time series with those from SeaWiFS, Aqua MODIS, and Terra MODIS shows that the scatter in the VIIRS lunar observations is consistent with that observed for the heritage instruments. Based on these analyses, the VOST has derived a calibration lookup table for VIIRS ocean color data based on fits to the solar calibration time series.
\end{abstract}

Keywords: VIIRS, solar calibration, lunar calibration, cross calibration, radiometric stability, reflective solar bands

\section{INTRODUCTION}

The Suomi National Polar-orbiting Partnership (SNPP) Visible Infrared Imaging Radiometer Suite (VIIRS) was launched into an 824-km sun-synchronous polar orbit with a node of 13:30 on October 28, 2011. VIIRS acquired its first image when its nadir door was opened on November 21, 2011. The instrument's cooled focal planes were turned on January 20, 2012. VIIRS is a 22-band scanning filter radiometer whose design heritage is SeaWiFS (rotating telescope with half-angle mirror) and MODIS (focal plan layout, solar diffuser, solar diffuser screen, solar diffuser stability monitor). The primary calibration responsibility for VIIRS resides with the NOAA NESDIS/STAR VIIRS Sensor Data Record (SDR) Calibration Working Group (CWG). Since production of climate quality ocean color data products places stringent requirements on the on-orbit calibration of the reflective solar bands ${ }^{1}$ (see Fig. 1), the NASA VIIRS Ocean Science Team (VOST) has been implementing an in-house VIIRS on-orbit calibration capability to meet the ocean color requirements. Accordingly, this paper discusses on-orbit calibration of the VIIRS reflective solar bands (whose characteristics are listed in Table 1), specifically the calibration of the visible and near-infrared bands M1-M7. This paper presents the current status of the VOST calibration efforts, which are being carried out in cooperation with the NASA VIIRS Characterization Support Team (VCST), the USGS Robotic Lunar Observatory (ROLO) program, and the NESDIS/STAR SDR CWG. This work is a continuation of VOST efforts reported previously. ${ }^{2}$

Early in the VIIRS on-orbit operations, the Near-Infrared Degradation Anomaly was observed, where tungsten oxide contamination of mirrors in the rotating telescope assembly was found to be causing a rapid decrease in mirror reflectance for the near infrared to short wave spectral region. ${ }^{3,4}$ The operational configuration of VIIRS

Further author information:

R.E.E.: Robert.E.Eplee@nasa.gov, 3012860953 


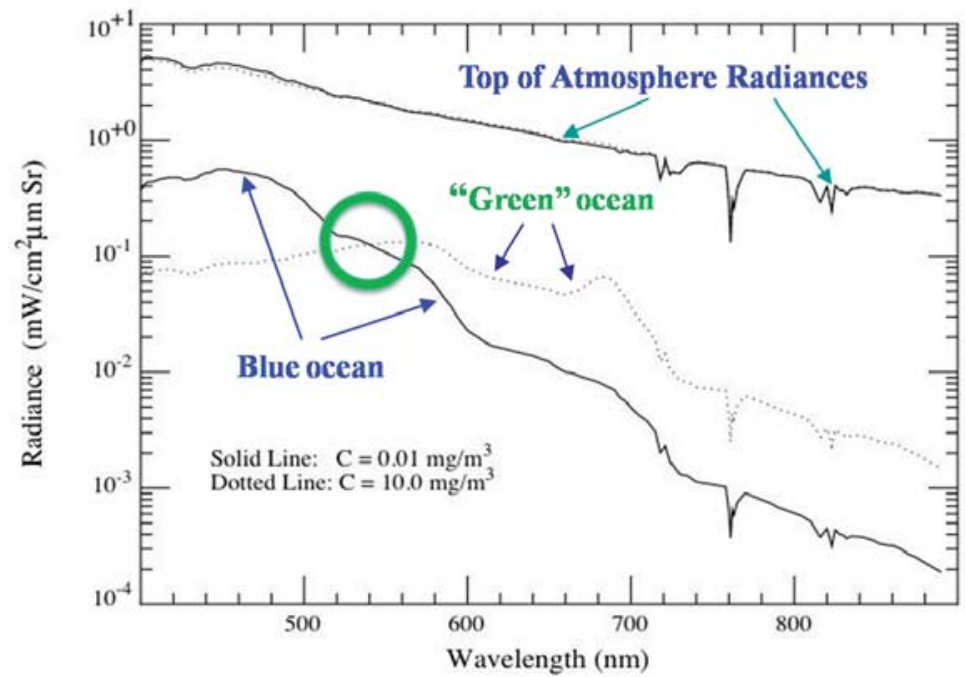

Figure 1. VIIRS Atmospheric Correction Rationale. Atmospheric correction removes $>85 \%$ of the signal from the top-of-the-atmosphere radiances, so a $0.1 \%$ calibration error in the TOA radiances will introduce an error of $\sim 1 \%$ in the water-leaving radiances. The green circle denotes the wavelength of the cross-over point between the blue-water and green-water spectra.

Table 1. VIIRS Reflective Solar Bands. The SWIR focal plane was not turned on until January 20, 2012. This paper addresses the calibration of bands M1-M7.

\begin{tabular}{||c|c|c|c|c|c|c||}
\hline Band & $\begin{array}{c}\text { SDSM } \\
\text { Band }\end{array}$ & $\begin{array}{c}\text { Band Center } \\
(\mathbf{n m})\end{array}$ & $\begin{array}{c}\text { Bandwidth } \\
(\mathbf{n m})\end{array}$ & $\begin{array}{c}\text { Single/Dual } \\
\text { Gain }\end{array}$ & $\begin{array}{c}\text { Spatial Resolution } \\
\text { at Nadir }(\mathbf{m})\end{array}$ & $\begin{array}{c}\text { Focal } \\
\text { Plane }\end{array}$ \\
\hline \hline M1 & C1 & 412 & 20 & DG & 750 & VNIR \\
\hline M2 & C2 & 445 & 18 & DG & 750 & VNIR \\
\hline M3 & C3 & 488 & 20 & DG & 750 & VNIR \\
\hline M4 & C4 & 555 & 20 & DG & 750 & VNIR \\
\hline I1 & & 640 & 80 & SG & 375 & VNIR \\
\hline M5 & C5 & 672 & 20 & DG & 750 & VNIR \\
\hline M6 & C6 & 746 & 15 & SG & 750 & VNIR \\
\hline I2 & & 865 & 41 & SG & 375 & VNIR \\
M7 & C7 & 865 & 41 & DG & 750 & VNIR \\
\hline M8 & & 1240 & 20 & SG & 750 & SWIR \\
\hline M9 & & 1378 & 15 & SG & 750 & SWIR \\
\hline I3 & & 1610 & 60 & SG & 375 & SWIR \\
M10 & & 1610 & 60 & SG & 750 & SWIR \\
\hline M11 & & 2250 & 50 & SG & 750 & SWIR \\
\hline
\end{tabular}


was changed frequently while the anomaly was under investigation. Continuous operation of the instrument began on January 2, 2012 in preparation for the first lunar calibration, and the instrument operations have been stable since that time. The end result of the VOST on-orbit calibration analyses is the generation of a calibration lookup table for use in ocean data processing beginning with the start of continuous operations on January 2, 2012.

Solar calibration is the first method for monitoring the on-orbit radiometric performance of the reflective solar bands. To yield the actual change in instrument response, the solar diffuser (SD) time series must be corrected by the solar diffuser stability monitor (SDSM)-derived change in the bidirectional reflectance distribution function (BRDF) of the SD. The VIIRS SD is a Spectralon panel placed behind a solar attenuation screen; the combined screen transmission function and solar diffuser BRDF is $\sim 13 \%$. The SDSM is an 8-channel radiometer with wavelengths corresponding to VIIRS bands M1-M7 and a reference channel at $935 \mathrm{~nm}$. The SDSM views the solar diffuser (the combined SD BRDF and SD screen transmission is $\sim 1 \%$ ) and views the Sun through an attenuation screen that has $\sim 4 \%$ transmission. VIIRS observes sunlight reflected by the solar SD once per orbit as the spacecraft crosses the North Pole, moving from the Earth's shadow into sunlight. The SDSM sequentially observes the sun and the solar diffuser to monitor changes in the diffuser BRDF over time. In July, 2012 the frequency of SDSM measurements was reduced from once per solar calibration to once per day. The change in radiometric sensitivity over time is computed from the solar diffuser data (the F-factor), corrected for the change in the diffuser BRDF (the H-factor). This methodology yields a calibration of the instrument on a per-band, per-detector basis for high and low gain states and the two sides of the half-angle mirror.

Lunar calibration is the second method for monitoring the radiometric response of the reflective solar bands on orbit. The spacecraft is rolled once per month to observe the Moon through the Space View at a nominal phase angle of $51^{\circ}$. The resulting lunar images are processed into disk-integrated lunar irradiances, which are then compared with the USGS Robotic Lunar Observatory (ROLO) photometric model of the Moon ${ }^{5,6}$ to yield a time series of instrument measurements to model predictions, which represent the radiometric response of the instrument over time. This methodology yields a calibration of the instrument on a per-band basis for the two sides of the half angle mirror. Changes in the lunar observing geometry over the course of a year limit the number of lunar observations to 8-9 per year.

At this point in the VIIRS mission, the VOST uses a detailed comparison of solar and lunar calibrations to minimize the uncertainties in the instrument response over time. The relative trends in radiometric response for VIIRS in the solar data and lunar data are generally in agreement, though to date there have only been 13 scheduled and 4 serendipitous lunar calibrations. Consequently, the lunar data is being used to validate the radiometric trends observed in the solar calibrations. The VIIRS lunar observations to date have been compared with the lunar observations of SeaWiFS, Aqua MODIS, and Terra MODIS as a means of assessing the radiometric performance of VIIRS with respect to these heritage instruments. As more lunar calibration data become available the lunar time series may supplement or supplant the solar calibration data as the primary radiometric monitor.

\section{SOLAR CALIBRATIONS}

VIIRS views sunlight reflecting off of the solar diffuser as the NPP spacecraft passes over the North Pole, moving from the Earth's shadow into the Sun. Fig. 2 shows a radiance profile across the diffuser during a typical solar calibration, a radiance profile observed by the solar diffuser stability monitor for the same calibration, and an image of the solar diffuser as observed by band M4. VIIRS solar diffuser observations provide the monitor for the radiometric response of the instrument, while the ratio of SDSM diffuser observations to SDSM solar observations provide the monitor for the diffuser BRDF.

\subsection{Solar Diffuser Stability Monitor Trends}

The BRDF history function ${ }^{2}$ is the ratio of the solar diffuser measurement to the direct solar measurement:

$$
h(\lambda, t)=\frac{d n_{s d}(\lambda, t)}{d n_{\text {sun }}(\lambda, t)} \frac{\cos (\phi(t)) \tau_{s d s m}(\lambda)}{\tau_{s d s}(\lambda) B R D F\left(\lambda, t_{o}\right) \Omega_{s d s m}}
$$

where: 

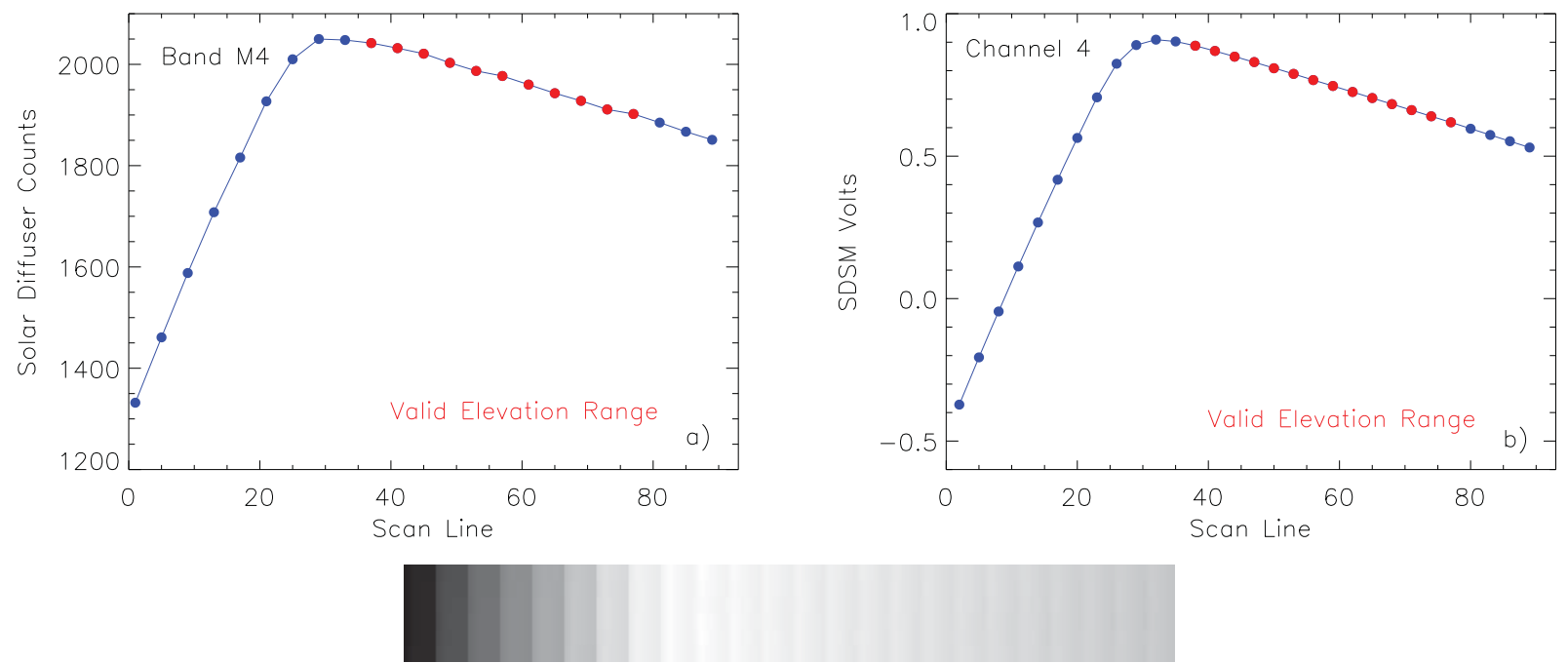

Figure 2. Solar Diffuser Calibration Profile. a) VIIRS band M4 profile in diffuser counts. b) SDSM channel 4 profile in SDSM volts. Red data points occur within the range of elevation angles where the BRDF of the diffuser and the transmission function of the solar diffuser and the SDSM screens were measured. Observations for each calibration are averaged over these valid scans. c) Solar diffuser image for band M4.

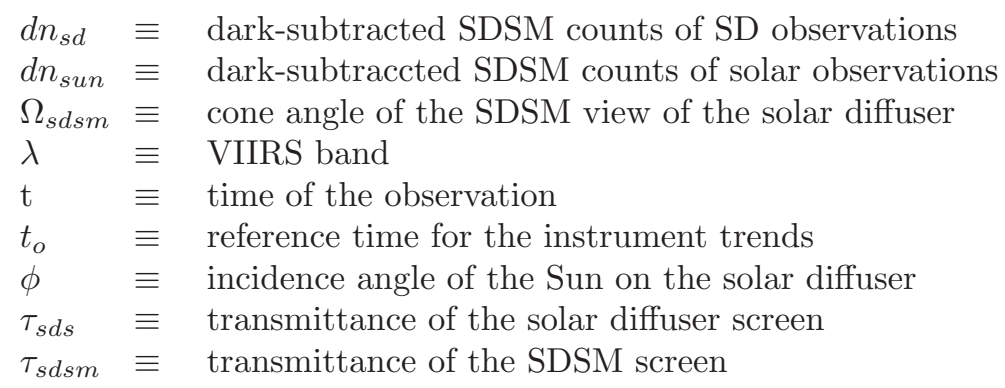

In these computations, the VOST uses the latest screen transmission functions and diffuser BRDF measurements from $\operatorname{VCST} .^{7,8}$ The time-dependent BRDF correction, the H-factor, is:

$$
H(\lambda, t)=\frac{h(\lambda, t)}{h\left(\lambda, t_{0}\right)}
$$

The SDSM trends over time are subject to measurement noise and other instrumental artifacts. The diffuser BRDF is assumed to be invarint with time at $935 \mathrm{~nm}$, so the SDSM reference channel at $935 \mathrm{~nm}$ is used to normalize the SDSM trends for these artifacts. ${ }^{9}$ The SDSM time series for channels $1-7$, normalized by the reference channel, are used to correct the band M1-M7 F-factors for changes in the diffuser BRDF. Fig. 3 shows the SDSM trends. The BRDF degradation decreases with increasing wavelength, as is expected. Since the SDSM measurements occur once per day since July 2012, the VOST interpolates the normalized H-factors for channels 1-7 to the time basis of the F-factors for bands M1-M7.

\subsection{Solar Diffuser Trends}

The VIIRS radiometric gain over time ${ }^{2}$ (the F-factor) is computed as the ratio of the predicted solar irradiance incident on the diffuser $L_{\text {pred }}$ to the measured radiance reflected by the diffuser $L_{s d}$ :

$$
F(\lambda, t)=R V S\left(\theta_{s d}, \lambda\right) \cos (\phi(t)) \frac{L_{p r e d}(\lambda, t)}{L_{s d}(\lambda, t)}
$$

where: 

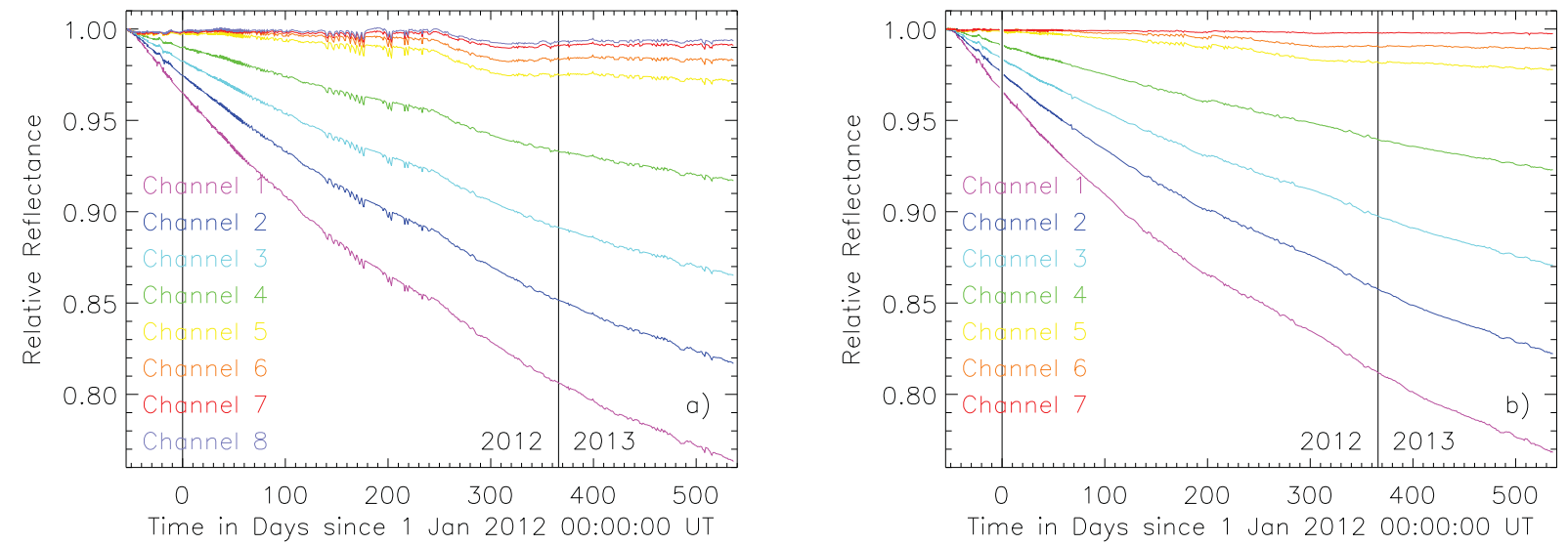

Figure 3. Solar Diffuser Stability Monitor Time Series. a) SDSM trends. b) SDSM trends after normalization of SDSM channel 8. The normalized series shows the change in diffuser BRDF over time. The H-factor time series is interpolated to the time basis of the solar calibration data.

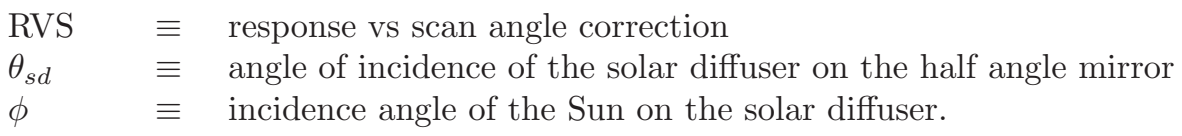

So, in terms of instrument measurements, the F-factor becomes:

$$
F(\lambda, t)=\frac{E_{\text {sun }}(\lambda)}{R_{\text {se }}^{2}(t)} \frac{R V S\left(\theta_{s d}, \lambda\right) \cos (\phi(t)) \tau_{s d s}(\lambda) B R D F(\lambda, t o)}{c_{0}(\lambda, t)+c_{1}(\lambda, t) d n_{s d}(\lambda, t)+c_{2}(\lambda, t) d n_{s d}^{2}(\lambda, t)} H(\lambda, t)
$$

where:

$$
\begin{array}{ll}
E_{\text {sun }} & \equiv \text { solar irradiance } \\
R_{\text {se }} & \equiv \text { Sun-Earth distance } \\
d n_{s d} \equiv \text { dark-subtracted instrument counts of SD observations } \\
c_{i} \equiv \text { instrument counts to radiance conversion coefficients }
\end{array}
$$

In these computations, the VOST uses the latest screen transmission functions and diffuser BRDF measurements from VCST. ${ }^{7,8}$ The inverse of the F-factor times series is the radiometric response over time. The F-factor time series for VIIRS are shown in Fig. 4.

The VOST constructs F-factor lookup tables for VIIRS from radiometric fits to the F-factor time series. Currently, the VOST fits the time series for bands M1-M7 with exponential plus linear functions of time:

$$
f(\lambda, t)=A_{0}(\lambda)-A_{1}(\lambda)\left[1-e^{-A_{2}(\lambda)\left(t-t_{0}\right)}\right]-A_{3}(\lambda)\left(t-t_{0}\right)
$$

where $A_{i}$ are the fitted values of the function. The fits to the F-factor time series, and the residuals of those fits, are shown in Fig. 5. The residuals for band M1-M4 are correlated, as are the residuals for bands M5-M7. The residuals show periodic signals, which most likely arise from errors in the the screen transmission and diffuser BRDF functions.

\section{LUNAR CALIBRATIONS}

The VIIRS lunar calibration methodology stems from MODIS heritage in that on an approximately monthly basis the NPP spacecraft is rolled to observe the Moon at a target phase angle through the space view. ${ }^{10}$ One difference from MODIS is that the VIIRS instrument design forces lunar observations to be obtained while the 

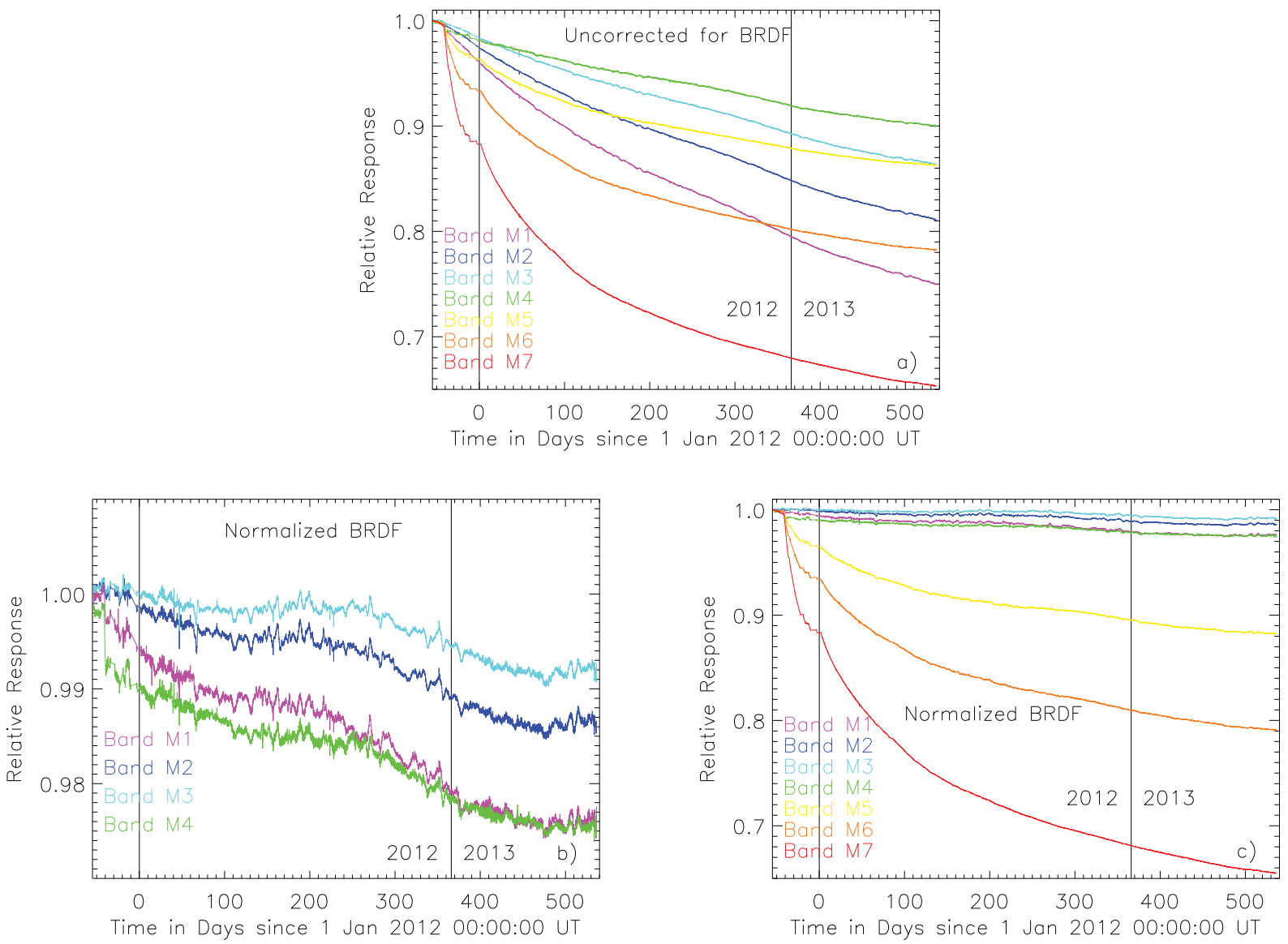

Figure 4. Solar Diffuser Time Series. a) The series for bands M1-M7, uncorrected for diffuser BRDF drift. b) The series for bands M1-M4, corrected for diffuser BRDF drift. c) The series for bands M1-M7, corrected for diffuser BRDF drift. 

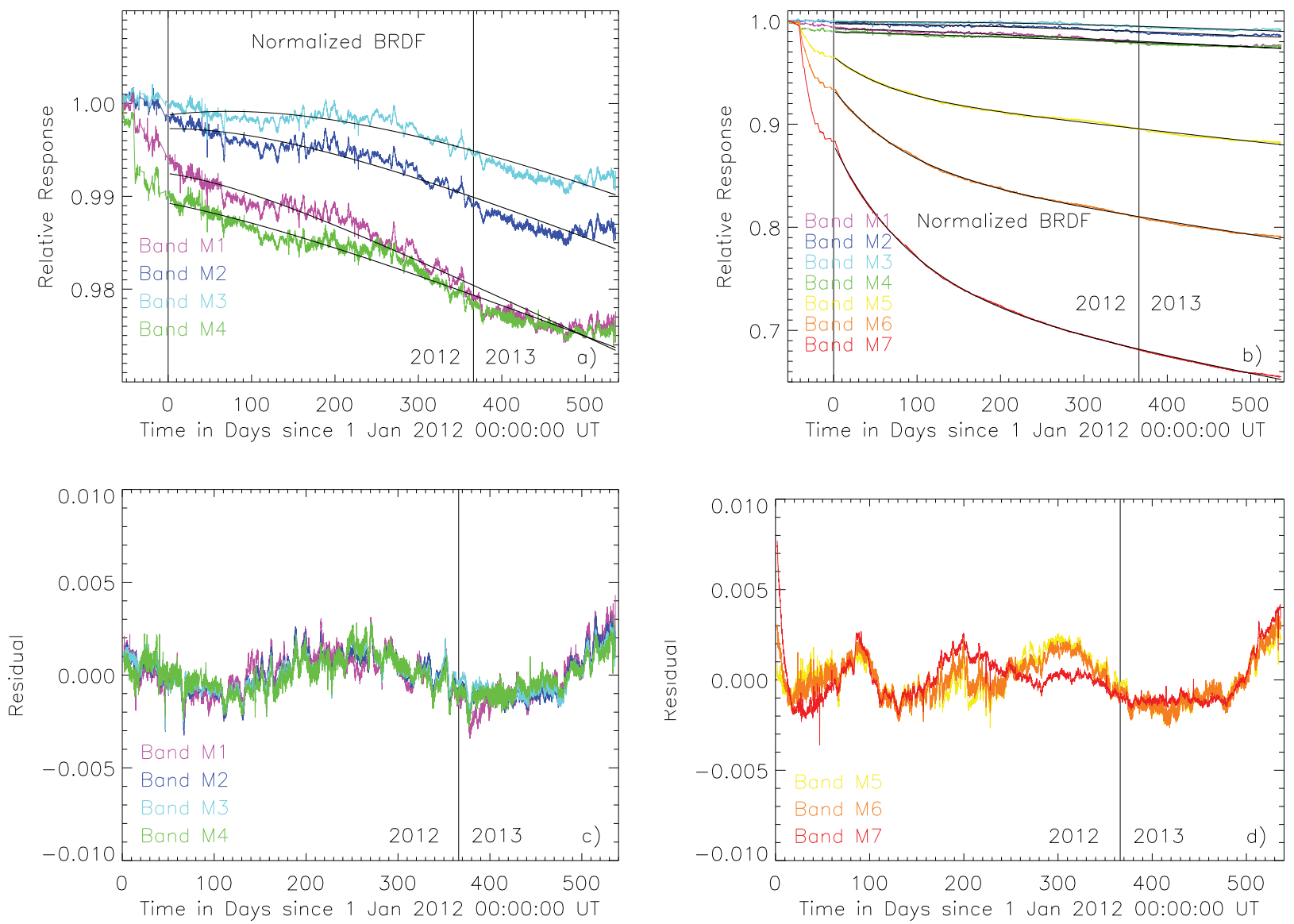

Figure 5. Fits to Solar Diffuser Time Series. Exponential plus linear functions of time are fit to the time series. a) The fits for bands M1-M4. b) The fits for bands M1-M7. c) Residuals of the fits for bands M1-M4. d) Residuals of the fits for bands M5-M7. 
Table 2. Lunar Calibrations. The VIIRS lunar calibrations obtained through June 2013.

\begin{tabular}{||c|c|c|c|c|c||}
\hline Cal Date & Number & Cal Type & Bands & Gains & Phase \\
\hline \hline 4 Jan 2012 & 1 & Roll & M3-M7 & hi,lo & -55.4 \\
\hline 5 Jan 2012 & 1 & Serendipitous & M1-M3 & hi,lo & -44.5 \\
\hline 3 Feb 2012 & 1 & Roll & M1-M11 & hi,lo & -56.8 \\
\hline 4 Mar 2012 & 1 & Serendipitous & M3, M5-M11 & hi,lo & -48.9 \\
\hline $\begin{array}{c}\text { 2 Apr 2012 - } \\
\text { 31 May 2012 }\end{array}$ & 3 & Roll / Sector & M1-M11 & hi & -51.7 \\
\hline 28 Jun 2012 & 1 & Serendipitous & M1-M11 & hi,lo & -65.7 \\
\hline $\begin{array}{c}\text { 25 Oct 2012- } \\
\text { 21 May 2013 }\end{array}$ & 8 & Roll / Sector & M1-M11 & hi & -50.8 \\
\hline 19 Jun 2013 & 1 & Serendipitous & M1-M4, M8,M9,M11 & hi,lo & -56.6 \\
\hline
\end{tabular}

spacecraft is in the sunlight. Accordingly, possible observing geometries require spacecraft roll angles of $14^{\circ}$ or less. The VIIRS methodology for observing the Moon has evolved to meet the on-orbit performance of the spacecraft:

1) The initial set of observations were made using the space view sector of the scan line data collection. VIIRS bands are not co-registered in the space view sector, so the size of the VIIRS visible/near infrared and short wave infrared focal planes, compared to the 48 sample angular extent of the space view, prevent all of the reflective solar bands fully observing the Moon in the space view at a single roll angle. Implemented for the January 4, 2012 observations.

2) The first alternative approach to observing the Moon with all 14 reflective solar bands was to perform lunar roll maneuvers on two successive orbits at roll angles selected to target different sides of the focal planes. The drawback to this approach is the operational impact of doubling the number of spacecraft maneuvers. Implemented for the February 3, 2012 observations.

3) The second alternative approach was to perform a shift of the scan line data collection so that the earth view sector, where band co-registration takes place, was rotated to cover the space view. This approach allowed all 14 solar reflective bands to view the Moon during a single lunar roll maneuver. Normally, the earth view sector allows the detector gain to be set automatically by the pixel brightness. To avoid lunar images with pixels in mixed gain states, the instrument is commanded to fixed high gain. Implemented for the April 2, 2012 observations, and to be continued for the foreseeable future.

4) The initial target phase angle has been adjusted from $55^{\circ}$ to $51^{\circ}$ to meet thermal exclusion zone requirements. Implemented for the April 2, 2012 observations, and to be continued for the foreseeable future.

As was the case with MODIS, the Moon occasionally moves through the space view as the spacecraft/Moon geometries coincide, though the phase angle at these times is larger than the target phase angle. The VOST uses these serendipitous lunar observations to validate the planned lunar observations. The serendipitous observations may not provide full disk lunar images for all of the bands because of the limited size of the space view. A shortcoming to the VIIRS lunar calibration strategy is that for approximately three months out of the year (normally during the northern hemisphere summer) the Moon is below the Earth's horizon during lunar calibration opportunities. ${ }^{10}$ Such annual gaps in lunar observations also occur for Terra MODIS and Aqua MODIS. The next NPP VIIRS lunar calibration opportunity will be in October 2013. Table 2 provides the lunar calibrations obtained so far by VIIRS and used in this analysis.

During a typical lunar maneuver, the spacecraft is rolled to observe the Moon at the target phase angle, the Moon drifts through the space view due to orbital motion of the spacecraft, then the spacecraft is rolled 
back to nadir. Fig. 6 shows the sequence of lunar images observed by band M4 during the May 2, 2012 lunar calibration. The single gain bands are aggregated on orbit; at nadir the data from the single gain bands undergo a 3:1 aggregation along scan. Aggregation affects band M6, M8-M11, and the I bands. Fig. 6 also shows typical lunar images from the rotated earth view sector for bands M4 (unaggregated) and M6 (aggregated).

The ROLO model of the Moon requires disk-integrated lunar irradiances as input. Consequently, the VOST only uses full-disk images from the lunar calibration image sequence in computing band-averaged disk-integrated irradiances for each gain and mirror side for the calibration. The along-scan aggregation of the single-gain bands is incorporated into the oversampling correction applied to the lunar irradiances which are input to the ROLO model.

The VOST uses the ROLO model to normalize the lunar calibration time series for variations in observing geometry: Instrument/Moon distances, Sun/Moon distances, phase and libration angles. The ROLO model predicts the reflectances of the Moon based on the phase and libration angles of the observation, computes the solar irradiances for the specified instrument bands, converts the lunar reflectances to irradiances using the solar irradiances, then uses the time of the observation and the position of the spacecraft to normalize the lunar irradiances to the values as seen by the instrument. The radiometric output of the model is the ratio of the instrument measurement and model prediction:

$$
P(\lambda, t)=\frac{K_{d}(t)}{A_{\text {moon }}(\lambda)} \frac{E_{\text {Inst }}(\lambda, t)}{E_{\text {sun }}(\lambda)}-1=\frac{E_{\text {inst }}(\lambda, t)}{E_{\text {rolo }}(\lambda, t)}-1
$$

where:

$$
\begin{aligned}
& A_{\text {Moon }} \equiv \text { lunar reflectance predicted by the ROLO model } \\
& E_{\text {Sun }} \equiv \text { solar irradiance } \\
& E_{\text {rolo }} \equiv \text { lunar irradiance predicted by the ROLO model } \\
& K_{d} \equiv \text { Instrument/Moon and Sun/Moon distance corrections. }
\end{aligned}
$$

Instrument irradiance is computed from the instrument radiance and the instantaneous field of view (IFOV) of the instrument:

$$
E_{\text {inst }}(\lambda, t)=I F O V_{\text {along-scan }} I F O V_{\text {along-track }} L_{T}(\lambda, t)
$$

$$
\begin{array}{ll}
I F O V_{\text {along-scan }} & \equiv 0.3104 \text { mrad for dual-gain M bands } \\
\text { IFOV } & \equiv 0.9313 \text { mrads for single-gain M bands in the 3:1 aggregation zone near nadir } \\
\text { IFOV along-track } & \equiv 0.9313 \text { mrad for all bands } \\
L_{T} & \equiv \text { the disk-integrated lunar radiance. }
\end{array}
$$

Fig. 7 shows the relative responses normalized to the first lunar calibration. The lunar time series are fit by exponential plus linear functions of time, as shown in Fig. 8. This figure also plots the residuals of the fits, which show significant periodic residuals. The VOST examined a number of possible origins for these signals, including focal plane temperature effects, distance effects, and residual libration correlations. The ROLO model derives corrections for the libration of the lunar surface around the sub-solar and sub-spacecraft points. The sub-solar libration corrections are wavelength dependent, while the sub-spacecraft librations are independent of wavelength. Examination of the residual plots in Fig. 8 shows trends with wavelength in the magnitude of the residuals. The libration angles for the longitude and latitude of the sub-spacecraft points are shown in Fig. 9.

To assess possible residual libration correlations in the lunar time series, the VOST fit the lunar time series with the exponential plus linear functions of time and an additional linear function of the longitude of the sub-spacecraft point. The fit has the form of:

$$
f(\lambda, t, \delta)=A_{0}(\lambda)-A_{1}(\lambda)\left[1-e^{-A_{2}(\lambda)\left(t-t_{0}\right)}\right]-A_{3}(\lambda)\left(t-t_{0}\right)+A_{4}(\lambda, \delta)
$$

where: 


\section{1}
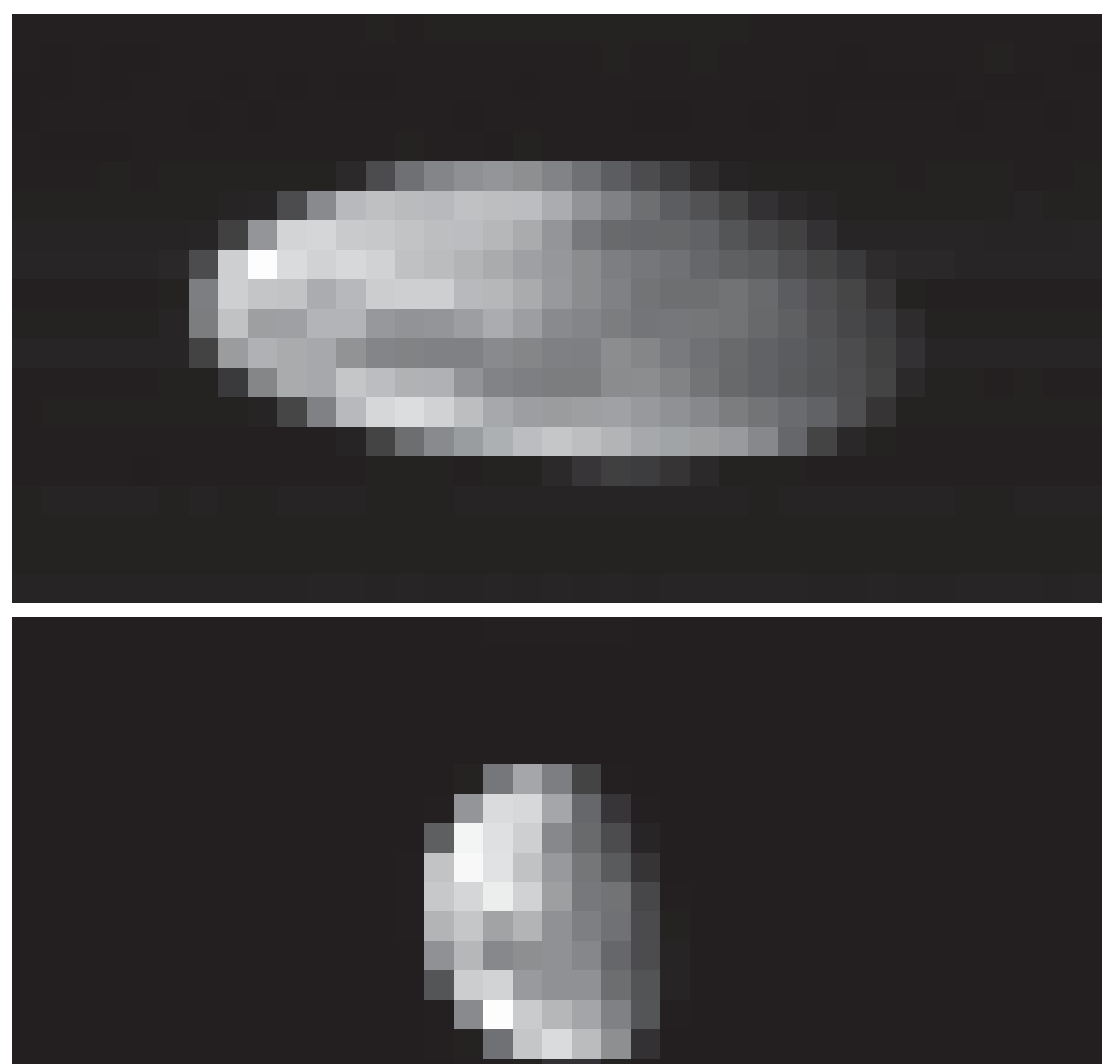

Figure 6. Lunar Calibration Image Sequence. a) Band M4 lunar sequence. b) Band M4 unaggregated full disk image. c) Band M6 aggregated full disk image. 

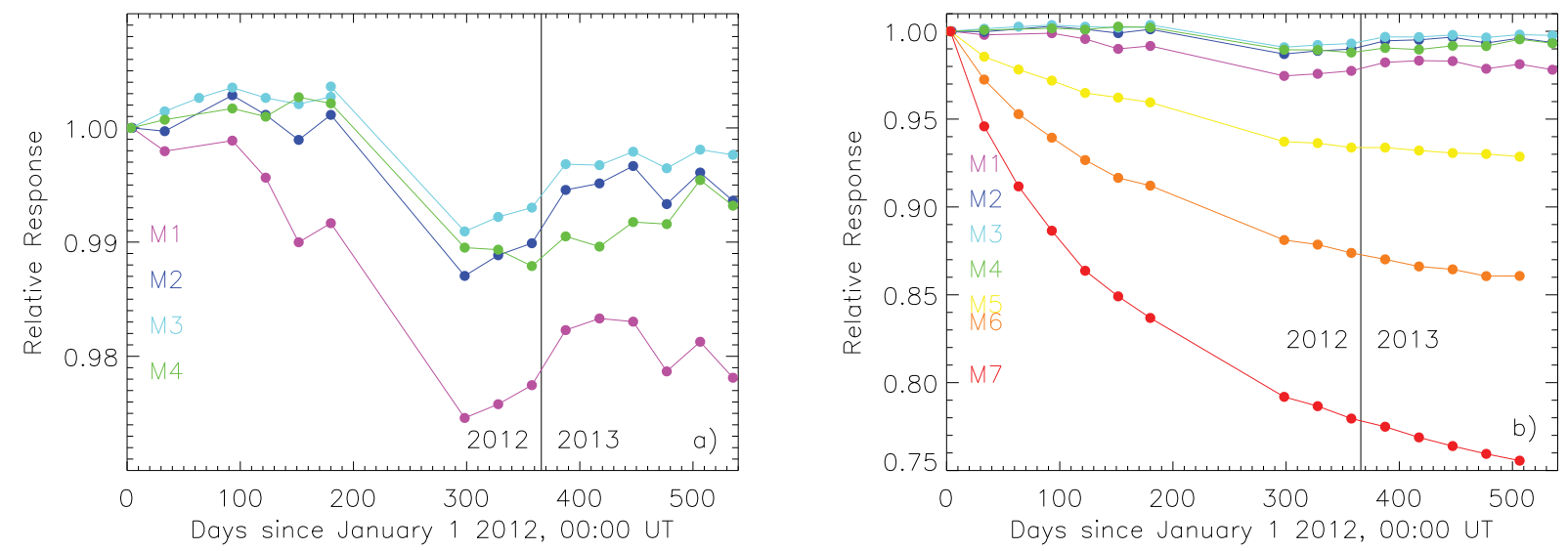

Figure 7. Lunar Calibration Time Series. a) The series for bands M1-M4. b) The series for bands M1-M7.

$A_{i} \equiv$ fitted values of the function

$\delta \equiv$ libration of the longitude of the sub-spacecraft point.

The lunar time series and fits to the time series are shown in Fig. 10. The periodic signals initially observed in the fit residuals are consistent with a residual libration correlation in the lunar time series. A comparison of the lunar residuals without and with the additional libration correction is shown in Fig. 11. The conclusion of this analysis is that while the ROLO model provides the primary, wavelength-independent sub-spacecraft point libration correction for the VIIRS lunar time series, the observations may have a residual libration effect that is wavelength dependent. The final determination that residual libration correlations are present in the lunar time series cannot be made until the lunar calibrations for October through December 2013 are in hand, which should show these correlations if they are present in the data.

Examination of the lunar residual plots (with additional libration correction) shows that there are no apparent periodic signals in the residuals. The noise appears to arise from observational scatter. The residuals for bands M1-M4 are correlated, as are the residuals for bands M5-M7, so there may be coherent noise in the residuals that can be corrected as was done for SeaWiFS, ${ }^{11}$ but this subject requires further analysis.

\section{COMPARISON OF SOLAR AND LUNAR CALIBRATIONS}

Solar calibrations and lunar calibrations provide two independent monitors of the radiometric response of VIIRS. Comparison of these two calibration trends provides insights into the uncertainties of both the solar and lunar data sets. Solar calibrations are performed once per orbit, while the lunar calibrations are performed once per month. To compare the two time series, the lunar time series for each band was normalized to a value that minimized the differences between the individual lunar calibrations and the closest (in time) solar calibrations over the mission. Fig. 12 shows this comparison of lunar and solar radiometric trends over time. The first two plots of the figure show the solar calibration time series, with the normalized lunar observations including the additional libration correction, superimposed. The third plot shows the differences between the lunar and solar time series at the time of the lunar observations, without the additional libration corrrection applied to the lunar data. The fourth plot shows the differences between the lunar and solar time series, with the additional libration correction applied to the lunar data. The fourth plot shows that the differences between the two calibration time series are small and that there are not any periodicities in the differences.

A further comparison of the solar and lunar calibration trends is afforded by comparing residuals of fits to the solar time series (Fig. 5) with residuals of fits to the lunar time series (Fig. 10). For both sets of time series, each band was fit by exponential plus linear functions of time. The lunar residuals have also had the residual libration effect removed. Examination of these plots shows that the residuals are correlated for bands M1-M4 

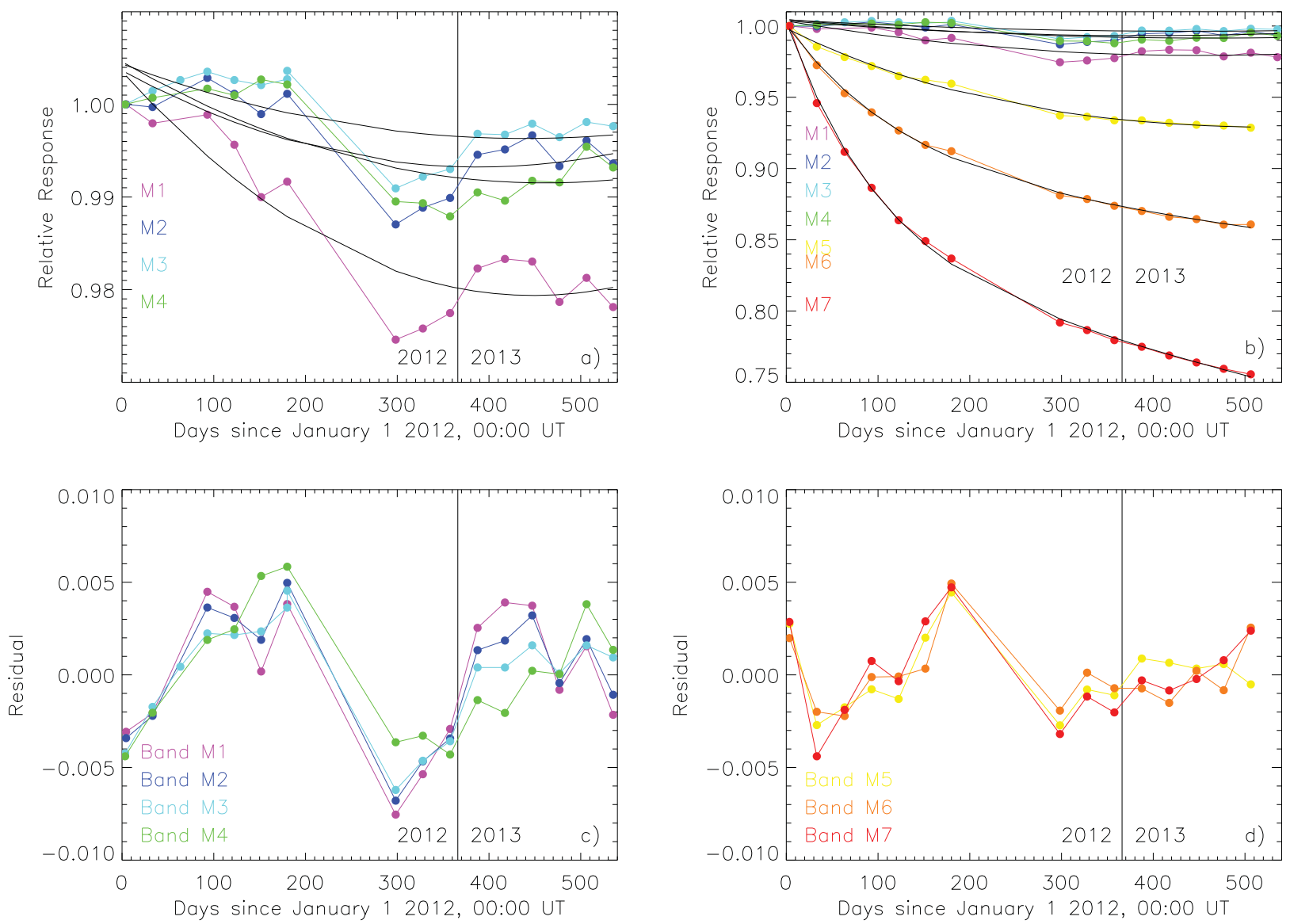

Figure 8. Fits to Lunar Calibration Time Series. Exponential plus linear functions of time are fit to the time series. a) The fits for bands M1-M4. b) The fits for bands M1-M7. c) Residuals of the fits for bands M1-M4. d) Residuals of the fits for bands M5-M7.

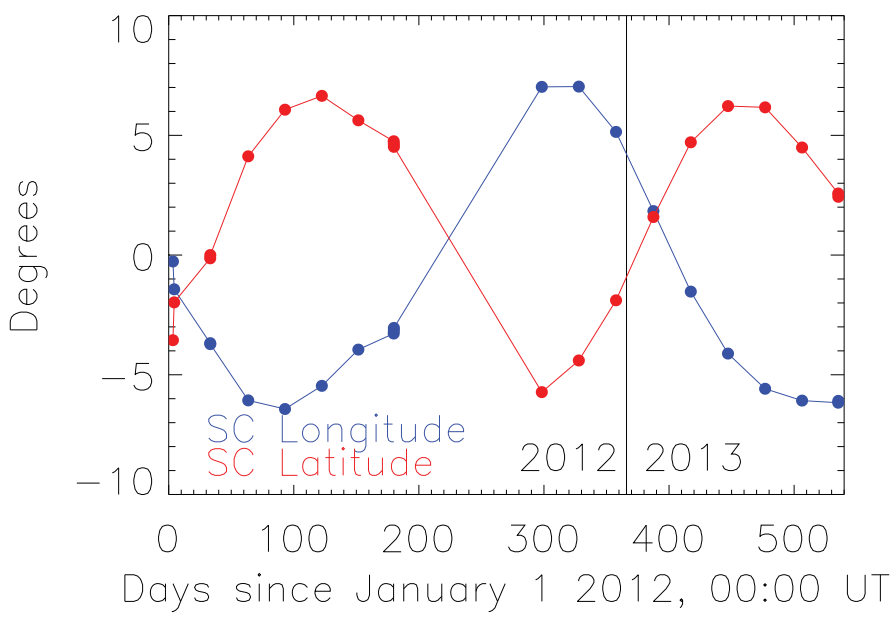

Figure 9. Sub-Spacecraft Lunar Librations. The time series of sub-spacecraft longitude and latitude libration angles are shown. 

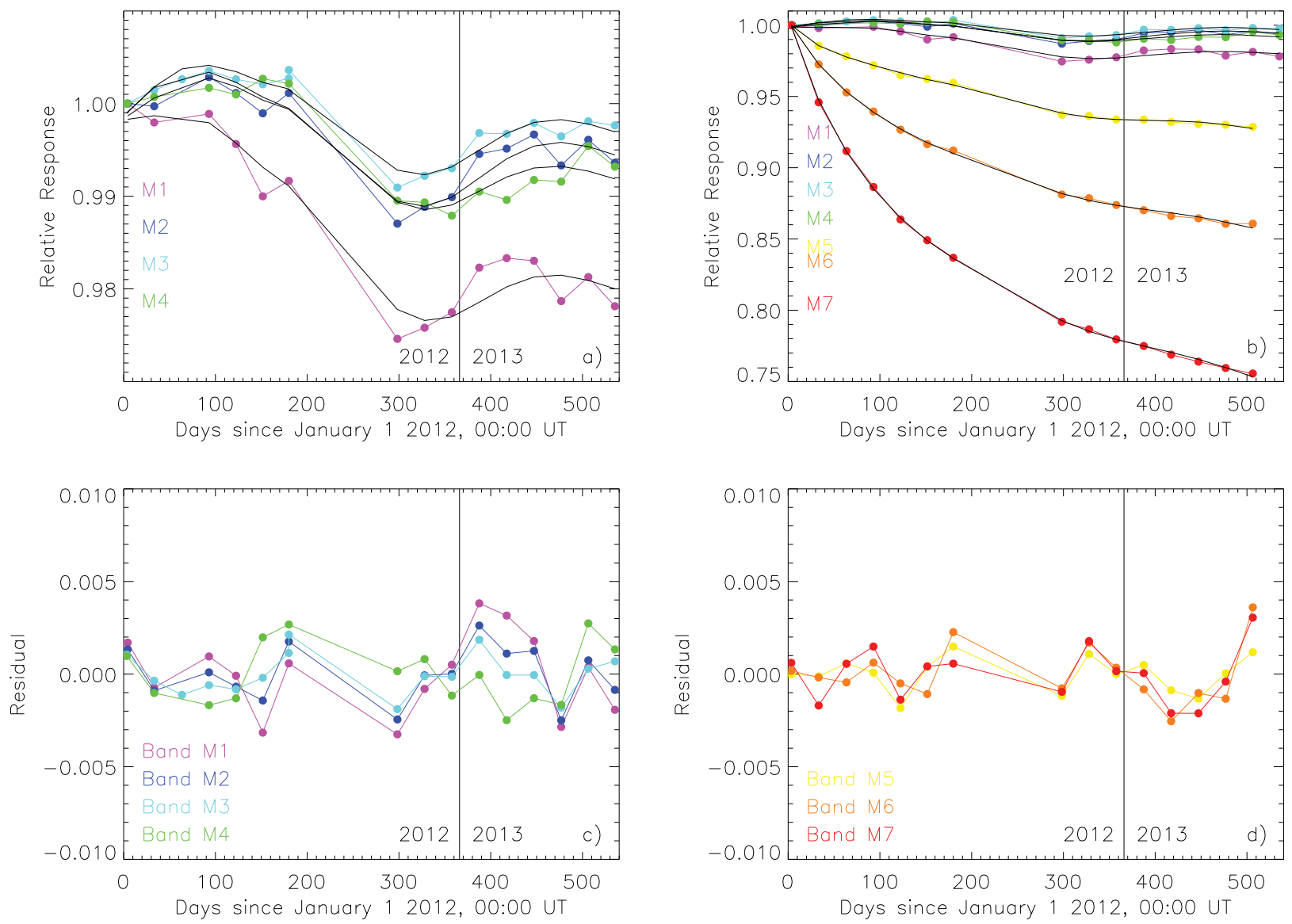

Figure 10. Fits to Lunar Calibration Time Series. Exponential plus linear functions of time and linear functions of sub-spacecraft longitude are fit to the time series. a) The fits for bands M1-M4. b) The fits for bands M1-M7. c) Residuals of the fits for bands M1-M4. d) Residuals of the fits for bands M5-M7. 

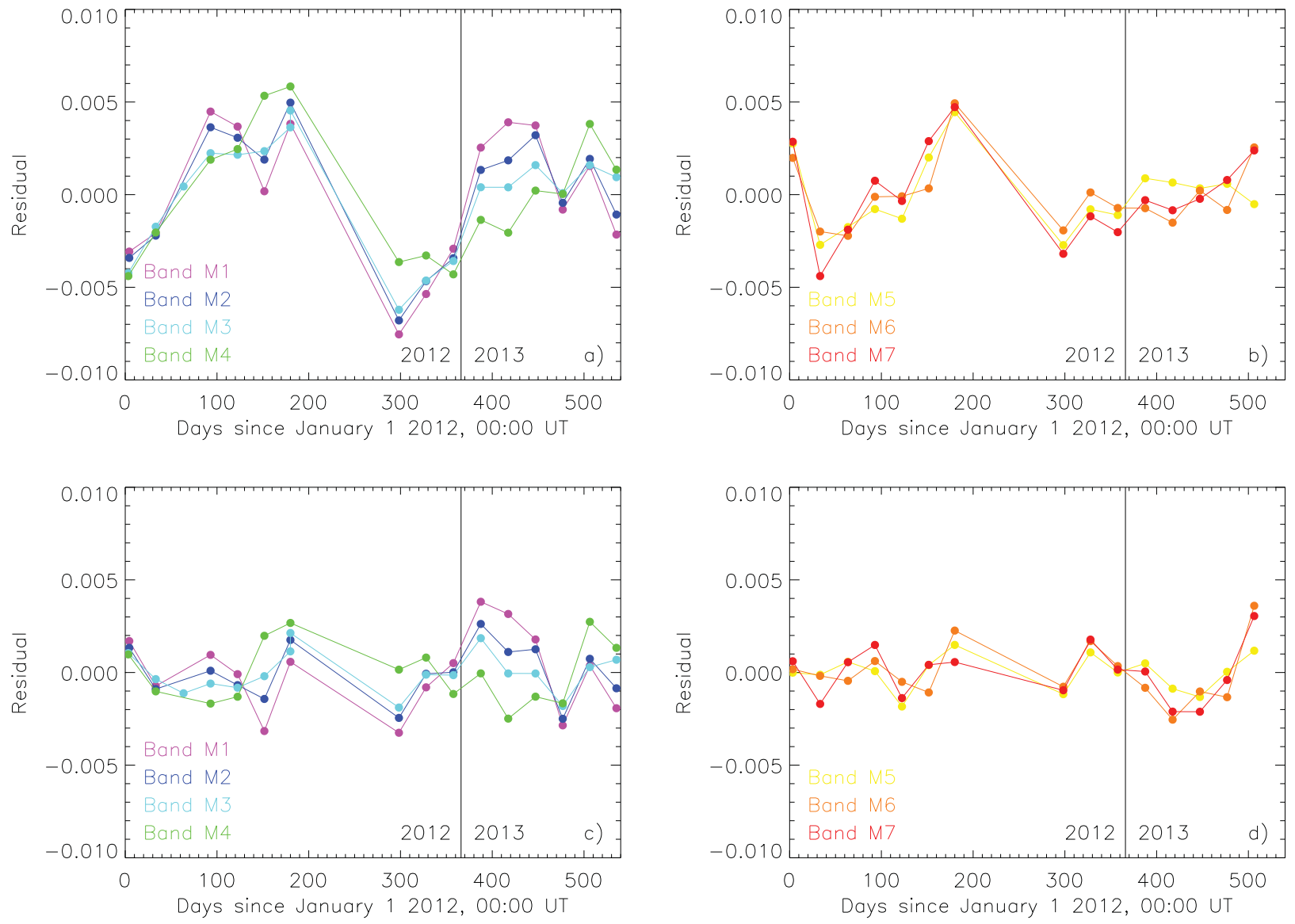

Figure 11. Lunar Calibration Residual Comparison. Residuals without and with the residual libration fit are compared. a) Residuals for bands M1-M4 without the libration fit. b) Residuals for bands M5-M7 without the libration fit. c) Residuals for bands M1-M4 with the libration fit. d) Residuals for bands M5-M7 with the libration fit. 

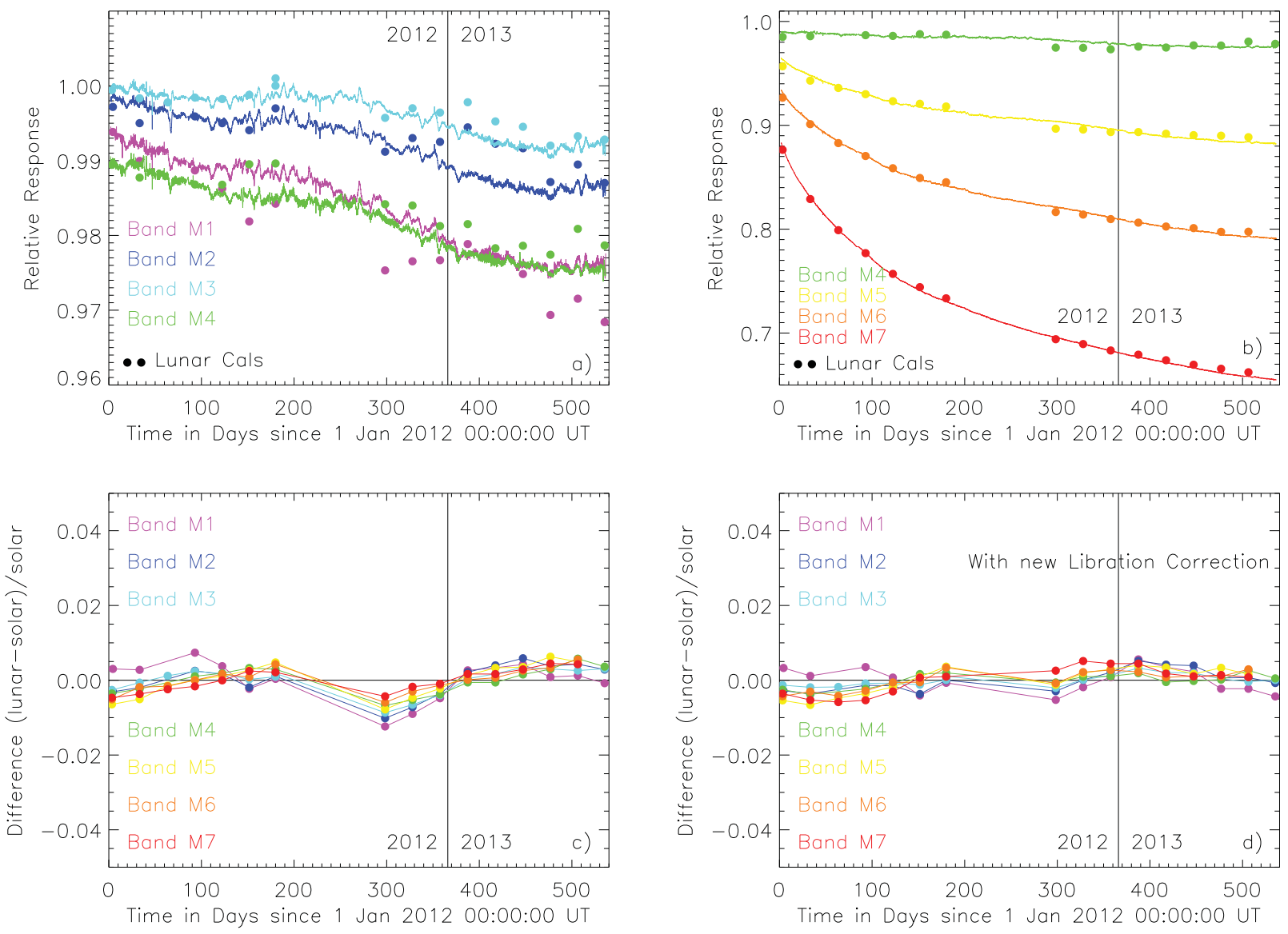

Figure 12. Solar and Lunar Calibration Comparison. a) Comparisons for bands M1-M4. b) Comparisons for bands M4-M7. c) Differences for bands M1-M7. d) Differences for bands M1-M7 with the additional libration correction applied to the lunar data. 
Table 3. On-Orbit Calibration Uncertainty. The relative uncertainties are the mean residuals for the solar and lunar calibrations. The solar / lunar calibration differences are means from the time series of (Fig. 12).

\begin{tabular}{||c|c||c|c||c||}
\hline Band & $\lambda \mathbf{( n m})$ & $\begin{array}{c}\text { Solar Cal } \\
\text { Uncertainty (\%) }\end{array}$ & $\begin{array}{c}\text { Lunar Cal } \\
\text { Uncertainty (\%) }\end{array}$ & $\begin{array}{c}\text { Relative } \\
\text { Differences (\%) }\end{array}$ \\
\hline \hline M1 & 412 & 0.0975 & 0.171 & 0.277 \\
\hline M2 & 445 & 0.0883 & 0.119 & 0.222 \\
\hline M3 & 488 & 0.0783 & 0.0841 & 0.120 \\
\hline M4 & 555 & 0.0810 & 0.142 & 0.143 \\
\hline M5 & 672 & 0.100 & 0.0711 & 0.312 \\
\hline M6 & 746 & 0.0962 & 0.116 & 0.217 \\
\hline M7 & 865 & 0.100 & 0.116 & 0.309 \\
\hline \hline
\end{tabular}

and again for bands M5-M7 for both the solar and lunar data sets. As noted in Section 2, the likely cause of the solar residuals is uncertainties in the screen transmission functions. As noted in Section 3, there are not any apparent periodicities in the lunar residuals. Comparison of the solar and lunar calibration time series have allowed the VOST to assess the uncertainties in the VIIRS on-orbit calibration, as summarized in Table 3 . The solar calibration time series shows uncertainties per band of $0.078-0.10 \%$, the lunar calibration time series shows uncertainties per band of $0.071-0.17 \%$, and the relative differences in the two on-orbit calibrations are $0.12-0.31 \%$. These uncertainties are comparable to the mission-long uncertainties of $0.033-0.13 \%$ determined for SeaWiFS ${ }^{11}$ and to the mission-long uncertainties of $0.38-0.94 \%$ determined for Terra MODIS lunar observations ${ }^{12}$ and of $0.30-0.58 \%$ determined for Aqua MODIS lunar observations. ${ }^{12}$

\section{LUNAR CALIBRATION INTERCOMPARISON}

Observations of the Moon provide a unique way of intercomparing the radiometric performance of two or more remote sensing satellite instruments on orbit. The latest on-orbit calibrations are applied to the lunar data to correct for radiometric drifts, thus allowing comparisons to be made with stable lunar irradiances. Use of the ROLO model for the lunar intercomparison removes the requirement of contemporaneous instrument operational lifetimes, aiding in the production of consistently calibrated data sets from these instruments. This intercomparison technique, which has previously been applied ${ }^{12}$ to SeaWiFS, Terra MODIS, and Aqua MODIS, is extended here to include VIIRS observations. The intercomparison includes all eight SeaWiFS bands, the MODIS visible and near-infrared bands which do not saturate on the Moon, and VIIRS bands M1-M7, as shown in Table 4.

The intercomparison over wavelength measures the calibration bias between the individual instruments and the ROLO model and allows the on-orbit calibration biases between the instruments to be determined. The input for this analysis is the mission-long average of the ROLO residuals for the primary lunar calibration data set for each instrument:

1) The monthly observations for SeaWiFS $\left(-7^{\circ},+7^{\circ}\right.$ phase),

2) The scheduled observations for Terra MODIS ( $+55^{\circ}$ phase),

3) The scheduled observations for Aqua MODIS ( $-55^{\circ}$ phase),

4) The scheduled observations for VIIRS $\left(-51^{\circ}\right.$ phase). 
Table 4. Bands for Lunar Intercomparison.

\begin{tabular}{||c|c||c|c||c|c||}
\hline \hline SeaWiFS & $\begin{array}{c}\text { Wavelength } \\
(\mathbf{n m})\end{array}$ & MODIS & $\begin{array}{c}\text { Wavelength } \\
(\mathbf{n m})\end{array}$ & VIIRS & $\begin{array}{c}\text { Wavelength } \\
(\mathbf{n m})\end{array}$ \\
\hline \hline B1 & 412 & B8 & 412 & M1 & 412 \\
\hline B2 & 443 & B9 & 442 & M2 & 445 \\
\hline & & B3 & 468 & & \\
\hline B3 & 490 & B10 & 487 & M3 & 488 \\
\hline B4 & 510 & B11 & 530 & & \\
\hline B5 & 555 & B12 & 547 & M4 & 555 \\
\hline B6 & 670 & B1 & 647 & M5 & 672 \\
\hline B7 & 765 & & & M6 & 748 \\
\hline B8 & 865 & B2 & 857 & M7 & 865 \\
\hline \hline
\end{tabular}

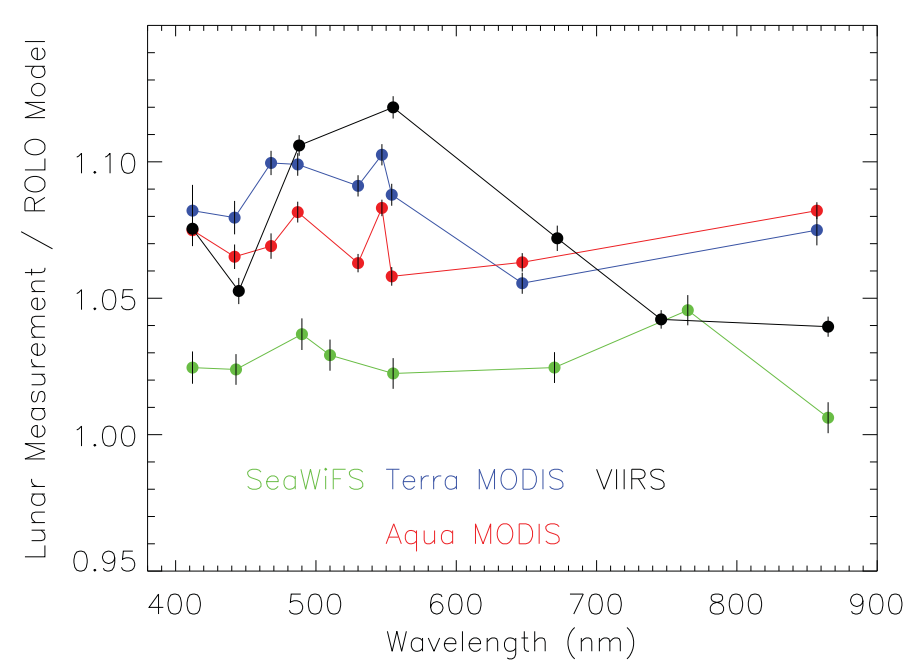

Figure 13. Lunar Intercomparison. The VIIRS calibration bias relative to the ROLO Model, compared to the biases of SeaWiFS, Aqua MODIS, and Terra MODIS.

For SeaWiFS, Terra MODIS, and Aqua MODIS, each instrument has the on-orbit calibration applied to the lunar data to correct for changes in the radiometric response of the instrument. For VIIRS, the solar calibration corresponding to each lunar observation is used to correct for radiometric response changes. The results of the intercomparison are shown in Fig. 13 and summarized in Table 5. The relative biases between the instrument are derived for Table 6 . The VIIRS calibration biases relative to the ROLO model are in family with those observed for the heritage instruments. ${ }^{11,12}$

The VOST has undertaken a comparison of the full set of SeaWiFS, MODIS, and VIIRS lunar observations. In addition to the scheduled calibrations, these data sets include the SeaWiFS high phase angle observations and the MODIS and VIIRS serendipitous observations. These data sets cover a phase angle range of $-85^{\circ}$ to $+85^{\circ}$. The intercomparison uses output from the ROLO model, without any coherent noise corrections being applied to the SeaWiFS or MODIS data and without the additional libration correction being applied to the VIIRS data. The intercomparison results for three bands (412 nm (blue), $555 \mathrm{~nm}$ (green), and $865 \mathrm{~nm}$ (near 
Table 5. Lunar Intercomparison. The biases of the instruments are computed relative to the ROLO model.

\begin{tabular}{||c|c|c|c||c|c|c|c||}
\hline \hline SeaWiFS & $\begin{array}{c}\text { Terra } \\
\text { MODIS }\end{array}$ & $\begin{array}{c}\text { Aqua } \\
\text { MODIS }\end{array}$ & VIIRS & $\begin{array}{c}\text { SeaWiFS / } \\
\text { ROLO }\end{array}$ & $\begin{array}{c}\text { TMODIS / } \\
\text { ROLO }\end{array}$ & $\begin{array}{c}\text { AMODIS / } \\
\text { ROLO }\end{array}$ & $\begin{array}{c}\text { VIIRS / } \\
\text { ROLO }\end{array}$ \\
\hline \hline B1 & B8 & B8 & M1 & $1.025 \pm 0.006$ & $1.082 \pm 0.009$ & $1.075 \pm 0.006$ & $1.075 \pm 0.005$ \\
\hline B2 & B9 & B9 & M2 & $1.024 \pm 0.006$ & $1.079 \pm 0.006$ & $1.065 \pm 0.004$ & $1.053 \pm 0.005$ \\
\hline & B3 & B3 & & & $1.100 \pm 0.004$ & $1.069 \pm 0.005$ & \\
\hline B3 & B10 & B10 & M3 & $1.037 \pm 0.006$ & $1.099 \pm 0.004$ & $1.082 \pm 0.004$ & $1.106 \pm 0.004$ \\
\hline B4 & B11 & B11 & & $1.029 \pm 0.006$ & $1.091 \pm 0.004$ & $1.063 \pm 0.003$ & \\
\hline B5 & B12 & B12 & M4 & $1.022 \pm 0.006$ & $1.103 \pm 0.004$ & $1.083 \pm 0.003$ & $1.120 \pm 0.004$ \\
& B4 & B4 & & & $1.088 \pm 0.004$ & $1.058 \pm 0.003$ & \\
\hline B6 & B1 & B1 & M5 & $1.025 \pm 0.006$ & $1.056 \pm 0.004$ & $1.063 \pm 0.004$ & $1.072 \pm 0.005$ \\
\hline B7 & & & M6 & $1.046 \pm 0.006$ & & & $1.042 \pm 0.003$ \\
\hline B8 & B2 & B2 & M7 & $1.006 \pm 0.006$ & $1.075 \pm 0.006$ & $1.082 \pm 0.003$ & $1.040 \pm 0.004$ \\
\hline \hline
\end{tabular}

Table 6. Lunar Relative Biases. The calibration biases among VIIRS, SeaWiFS, Terra MODIS, and Aqua MODIS are derived relative to the ROLO model, as shown in Fig. 13. The uncertainties are for the relative bias computations.

\begin{tabular}{||c|c|c||}
\hline $\begin{array}{c}\text { Intercomparison } \\
\text { Instruments }\end{array}$ & $\begin{array}{c}\text { Bias } \\
\mathbf{( \% )}\end{array}$ & $\begin{array}{c}\text { Uncertainty } \\
\mathbf{( \% )}\end{array}$ \\
\hline \hline SeaWiFS / Terra & $3-8$ & 1.4 \\
\hline SeaWiFS / Aqua & $3-8$ & 1.3 \\
\hline Terra / Aqua & $1-3$ & 1.3 \\
\hline SeaWiFS / VIIRS & $1-10$ & 1.3 \\
\hline Terra / VIIRS & $1-4$ & 1.3 \\
\hline Aqua / VIIRS & $1-6$ & 1.2 \\
\hline \hline
\end{tabular}



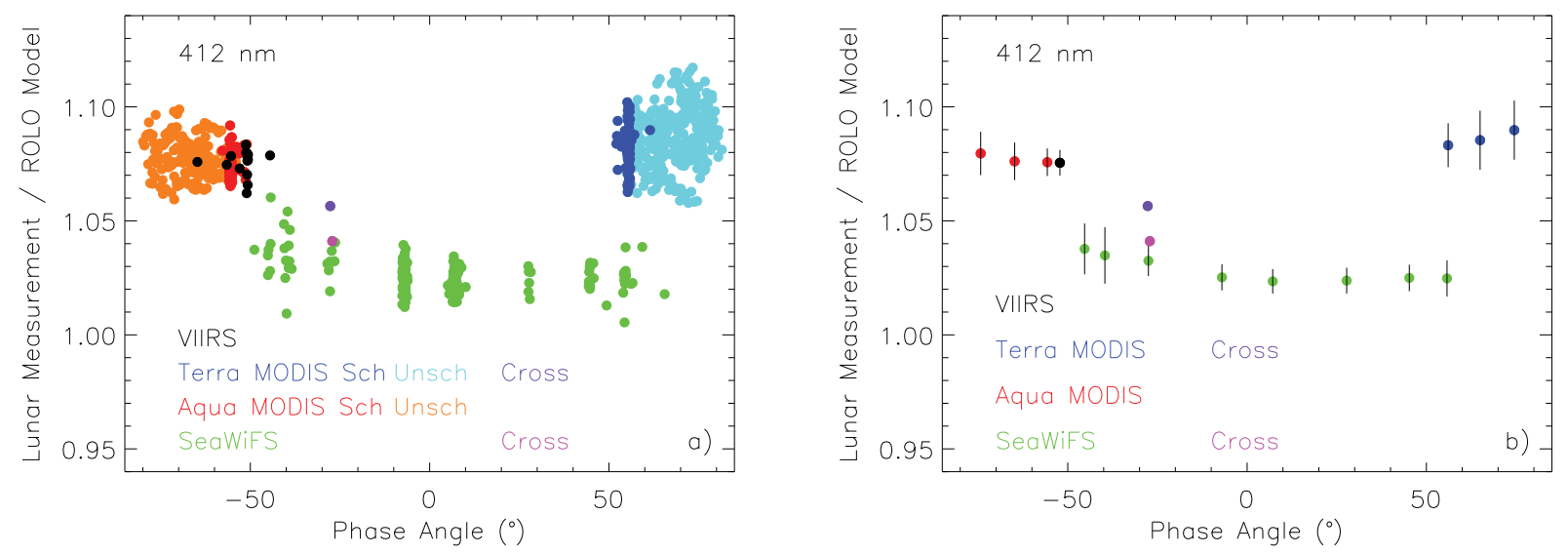

Figure 14. Lunar Intercomparison at 412 nm. a) VIIRS lunar residuals are compared with those for SeaWiFS and MODIS. b) VIIRS lunar residuals are consistent with heritage instruments.
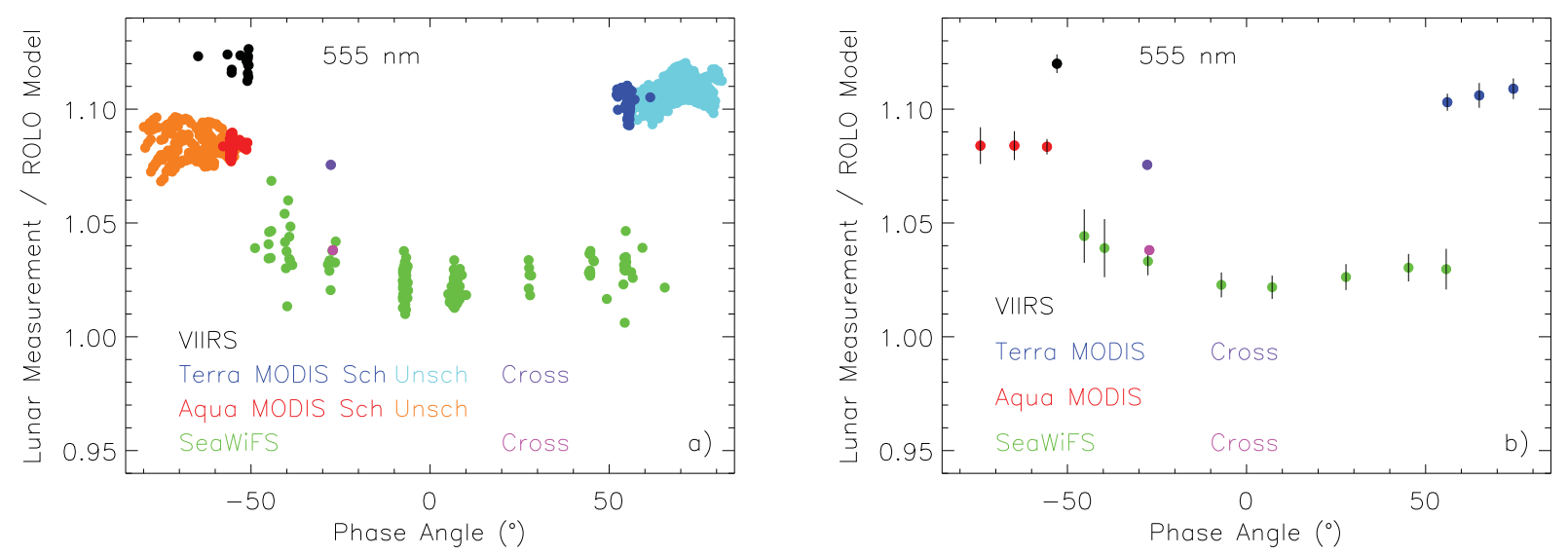

Figure 15. Lunar Intercomparison at $555 \mathbf{~ n m . ~ a ) ~ V I I R S ~ l u n a r ~ r e s i d u a l s ~ a r e ~ c o m p a r e d ~ w i t h ~ t h o s e ~ f o r ~ S e a W i F S ~ a n d ~}$ MODIS. b) VIIRS lunar residuals are consistent with heritage instruments.

infrared)) are shown in Fig. 14, Fig. 15, and Fig. 16. The second plot in each figure shows mean residuals for a number of phase angle ranges, which allow the significance of the variations as a function of phase angle to be examined. The magnitude of the observed scatter in the VIIRS lunar residuals is consistent with that observed for SeaWiFS, Terra MODIS, and Aqua MODIS. ${ }^{12}$

\section{CALIBRATION ASSESSMENT}

The VOST has developed two independent calibrations of the SNPP VIIRS moderate resolution reflective solar bands using solar diffuser and lunar observations through June 2013.

The solar calibration data have been processed using the most recent transmission screen and solar diffuser BRDF functions from VCST. The solar diffuser F-factor time series, corrected for changing diffuser BRDF with the SDSM-derived H-factor time series, are optimally fit with exponential plus linear functions of time to model the change in instrument response over time. Bands M1-M4 can be fit as well with linear functions of time, alone. The fits show mean residuals per band of $0.078-0.010 \%$. There are noticeable periodicities in the residuals, with correlations among bands M1-M4 and again among bands M5-M7. These residuals likely arise from errors in the screen transmission and diffuser BRDF functions. 

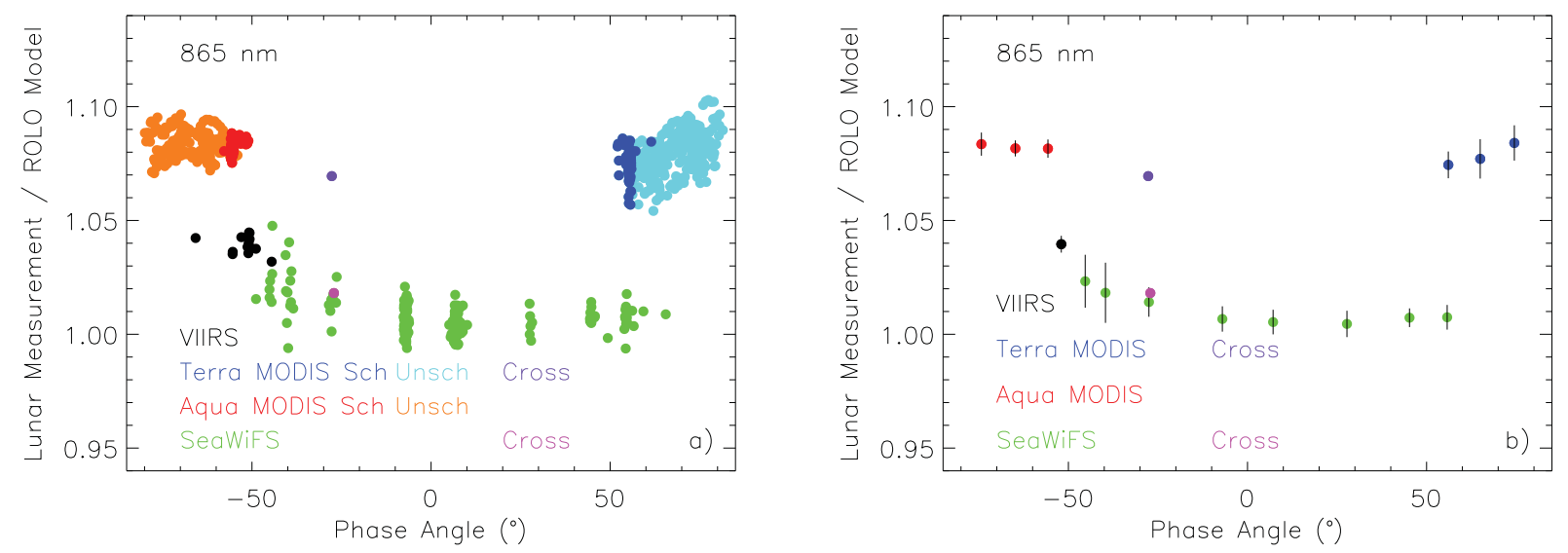

Figure 16. Lunar Intercomparison at $865 \mathbf{~ n m . ~ a ) ~ V I I R S ~ l u n a r ~ r e s i d u a l s ~ a r e ~ c o m p a r e d ~ w i t h ~ t h o s e ~ f o r ~ S e a W i F S ~ a n d ~}$ MODIS. b) VIIRS lunar residuals are consistent with heritage instruments.

The lunar calibration data have been processed using the ROLO photometric model of the Moon. There are apparent wavelength-dependent residual lunar libration correlations in the time series that are not accounted for by the ROLO model. Consequently, the lunar calibration time series are fit with exponential plus linear functions of time and with linear function of the sub-spacecraft libration angles to model the change in instrument response over time. The fits show mean residuals per band of $0.071-0.17 \%$. There are not any periodicities in the residuals, though correlations among bands M1-M4 and among bands M5-M7 are observed. Intercomparison of the VIIRS lunar time series with those from SeaWiFS, Aqua MODIS, and Terra MODIS shows that the scatter in the VIIRS lunar observations is consistent with that observed for the heritage instruments. Investigation of the residual wavelength-dependent libration effect in ROLO model output for VIIRS will continue with the resumption of lunar observations in October 2013, and will be undertaken using SeaWiFS and MODIS lunar calibration data.

Comparison of the solar and lunar time series shows that the relative differences in the two calibration are $0.12-0.31 \%$. The uncertainties in the VIIRS solar and lunar calibration time series are comparable to the mission-long uncertainties of $0.033-0.13 \%$ determined for SeaWiFS ${ }^{11}$ and to the mission-long uncertainties of $0.38-0.94 \%$ determined for Terra MODIS lunar observations ${ }^{12}$ and of $0.30-0.58 \%$ determined for Aqua MODIS lunar observations. ${ }^{12}$ Based on the solar and lunar calibration analyses, the VOST has derived a calibration lookup table for VIIRS derived from fits to the solar calibration time series.

The VIIRS on-orbit calibration time series (both solar and lunar) have uncertainties that are comparable to those for heritage instruments. Currently, based on SeaWiFS and MODIS experience, the VOST has achieved a radiometric stability for the VIIRS on-orbit calibration that is approaching the level required for production of ocean color climate data records from VIIRS data. The next year of on-orbit calibration data will affirm the current understanding of the VIIRS calibration.

\section{REFERENCES}

[1] National Research Council, Assessing Requirements for Sustained Ocean Color Research and Operations, The National Academies Press, Washington, D.C. (2011).

[2] R.E. Eplee, Jr., K.R. Turpie, G.F. Fireman, G. Meister, T.C. Stone, F.S. Patt, B.A. Franz, S.W. Bailey, W.D. Robinson, and C.R. McClain, "VIIRS on-orbit calibration for ocean color data processing," in Earth Observing Systems XVII, J.J. Butler, X. Xiong, and X. Gu, eds., Proc. SPIE 8510 85101G (2012).

[3] F. De Luccia, D. Moyer, E. Johnson, K. Rausch, N. Lei, K. Chiang, X. Xiong, J. Fulbright, E. Haas, and G. Iona, "Discovery and characterization of on-orbit degradation of the Visible Infrared Imaging Radiometer Suite (VIIRS) Rotating Telescope Assembly (RTA)," in Earth Observing Systems XVII, J.J. Butler, X. Xiong, and X. Gu, eds., Proc. SPIE 8510, 85101A (2012). 
[4] J.D. Barrie, P.D. Fuqua, M.J. Meshishnek, M.R. Ciofalo, C.T. Chu, J.A. Chaney, R.M. Moision, and L. Graziani, "Root cause determination of on-orbit degradation for the VIIRS Rotating Telescope Assembly," in Earth Observing Systems XVII, J.J. Butler, X. Xiong, and X. Gu, eds., Proc. SPIE 8510, 85101B (2012).

[5] H.H. Kieffer and T.C. Stone, "The spectral irradiance of the Moon," Astron. J. 129, 2887-2901 (2005).

[6] T.C. Stone and H.H. Kieffer, "Use of the Moon to support on-orbit sensor calibration for climate change measurements," in Earth Observing Systems XI, J.J. Butler and J. Xiong, eds., Proc. SPIE 6296, 62960Y (2006).

[7] J. McIntire, B. Efremova, D. Moyer, S. Lee, and X. Xiong, "Analysis of the Suomi-NPP VIIRS vignetting functions based on yaw maneuver data," in Earth Observing Systems XVII, J.J. Butler, X. Xiong, and X. Gu, eds., Proc. SPIE 8510, 85101K (2012).

[8] J. Fulbright, N. Lei, J. McIntire, B. Efremova, X. Chen, and X. Xiong, "Improving the characterization and performance of the Suomi-NPP/VIIRS solar diffuser stability monitor," in Earth Observing Systems XVIII, J.J. Butler, X. Xiong, and X. Gu, eds., Proc. SPIE 8866, 88661M (2013).

[9] J.P. Fulbright, N. Lei, K. Chiang, and X. Xiong, "Characterization and preformance of the Suomi-NPP VIIRS solar diffuser stability monitor," in Earth Observing Systems XVII, J.J. Butler, X. Xiong, and X. Gu, eds., em Proc. SPIE 8510, 851015 (2012).

[10] F.S. Patt, R.E. Eplee, R.A. Barnes, G. Meister, and J.J. Butler, "Use of the Moon as a calibration reference for NPP VIIRS," in Earth Observing Systems X, J.J. Butler, ed., Proc. SPIE 5882, 588215 (2005).

[11] R.E. Eplee, Jr., G. Meister, F.S. Patt, R.A. Barnes, S.W. Bailey, B.A. Franz, and C.R. McClain, "On-orbit calibration of SeaWiFS," Appl. Opt. 51, 8702-8730 (2012).

[12] R.E. Eplee, Jr., J.-Q. Sun, G. Meister, F.S. Patt, X. Xiong, and C.R. McClain, "Cross calibration of SeaWiFS and MODIS using on-orbit observations of the Moon," Appl. Opt. 50, 120-133 (2011). 